\title{
Analysis of Nutritional Constituents in Twenty Citrus Cultivars from the Mediterranean Area at Different Stages of Ripening
}

\author{
Almudena Bermejo*, Antonio Cano \\ Citriculture and Vegetable Production Department, Instituto Valenciano de Investigaciones Agrarias (IVIA), Valencia, Spain. \\ Email: "bermejo_alm@gva.es
}

Received February $16^{\text {th }}, 2012$; revised March $14^{\text {th }}, 2012$; accepted March $22^{\text {nd }}, 2012$

\begin{abstract}
Twenty Citrus cultivars grown in the Mediterranean climate were analysed at different stages of fruit maturity to determinate changes in organic acids, vitamin $\mathrm{C}$ and sugars. High-performance liquid chromatographic methods were used to identify and quantify of these compounds. The influences of variety, rootstocks and different stages of fruit maturity were observed. Generally, the vitamin C content was higher in varieties grafted on Troyer citrange that the corresponding cultivars grafted on Cleopatra mandarin. At commercial harvest stage, lemons, clementine mandarins and sweet oranges, showed the highest concentrations of vitamin C; citrons, limes and lemons, the higher amounts of organic acids; and mandarins and hybrids the highest amounts of sugars. Since sugars and acids played an important role in fruit flavor and their nature and concentration largely affect taste characteristic and organoleptic quality, we hope to relate genotypes and differences in final fruit quality. We have found clear differences in the content of sugars, ascorbic and organic acids for the different groups in agreement with the Citrus classification. Also climatic and cultural factors have affected to fruit quality, and anticipate or delay the collection generally results in a loss of bioactive compounds. The fruit quality was affected differently and we have observed differences in accordance with the rootstocks used but, the major differences in nutritional composition must be attributed mainly to genetic factors. The data presented are an important factor to chose varieties with a high potential as nutraceutical source.
\end{abstract}

Keywords: Citrus; Nutritional Quality; Vitamin C; Organic Acids; Sugars

\section{Introduction}

Citrus is one of the most important commercial fruit crops in the world, and fruit weight, size, acidity and maturity index, harvest time, chemical and nutritional composition are important quality traits for fresh citrus consumption and acceptance by the citrus industry. An increase in the consumption of fruits and vegetables is associated with a decrease in the incidence of cardiovascular disease and reduce risks of certain cancers. Thus, citrus fruits have received much attention because of its nutritional and antioxidant properties and nowadays prevention of health problems through nutrition is promoted intensively, due mainly to the contribution of antioxidant compounds including vitamin $\mathrm{C}$, phenolics compounds and carotenoids. Chemical variability of bioactive compounds and its relationship with genetic and climatic factors has been studied by diverse authors, and its contribution to the plant taxonomy has been reported [1-4]. Organic acids, sugars and phenolic compounds are among

${ }^{*}$ Corresponding author. the major compounds of citrus fruit pulp. Their nature and concentration largely affect taste characteristics and organoleptic quality. Organic acids and sugars vary accordig to species, varieties, and also environmental and horticultural conditions such as climate, rootstock, and irrigation [5]. Also the effect of citrus rootstocks on fruit nutricional quality has been studied by diverse authors. According to the kind of rootstock used, different morphological and biological characteristics are obtained, including plant growth and fruit production, tree size, adaptation to certain soil conditions, size, texture, internal quality and maturity $[6,7]$.

The content of vitamin $\mathrm{C}$ and other organic acids in fruits and vegetables can be influenced by various factors such as genotypic differences, preharvest climatic conditions and cultural practices, maturity and harvesting methods $[5,8,9]$. Organic acids are a useful index of authenticity in fruit products, since they have lower susceptibility to change during processing and storage than other components of fruits. At the same time, some or- 
ganic acids may be used as indicators of ripeness, bacterial activity and adulteration $[10,11]$. Previous reports of biochemical compounds have focused mainly on commercial varieties, and information regarding changes in biochemical constitutes of citrus fruit during ripening can be found in various reports $[12,13]$. However there is no comprehensive information regarding the changes in chemical bioconstituents during citrus fruits ripening in the same conditions of climate and field. We had studied the chemical variability of bioactive compounds in citrus pulp and juice and its relationship with genetic and climatic factors, and recently, we have evaluated the rind content of bioactive constituents (flavonoids, carotenoids, vitamin-C, essential oils and mineral composition) in several mandarin and orange cultivars from Mediterranean area [14-16]. Current paper provides significant new information to the citriculture industry to chose varieties with a high potential as nutraceutical source. Most of the studies on organic acid and sugar content are performed during fruit maturation but only a small amount data were obtained before maturation. In this way, Albertini et al. provided a wide report on sugar and organic acid accumulation during the early stages of fruit development in three citrus species (lemon, lime and orange) [5], while Pailly et al. evaluated the effects of harvest date on grapefruits [17]. More recently Ladaniya and Mahalle examined the fruit maturity changes in soluble sugars and organoleptic characteristics of "Mosambi" orange [18].

Maturity is one of the major factors that determines the compositional quality of fruits and vegetables [9]. From commercial point of view, it is important to extend the commercial harvest time of citrus fruits without compromising the nutricional quality. Now, the aim of our study was to better understand the behaviour of several citrus genotypes and characterize the changes in total vitamin $\mathrm{C}$, organic acids and carbohydrates during fruit maturation and, where possible, examine the influence of rootstocks on the bioactive content. Also the influence of variety on the content of some bioactive constituents during the best commercial harvest time having a goods balance of sweet taste and a refreshing aroma is discussed. Sugars are the major components of citrus juice soluble solids and sweetness of citrus juice is intrinsic to its sugar composition. Sucrose, fructose and glucose are the main sugars in citrus fruits. The main organic acids of citrus fruits are citric and malic acids. In addition, tartaric, benzoic, oxalic and succinic acids have been reported in smaller amounts [19]. Since these sugars and acids played an important role in fruit flavor and their nature and concentration largely affect taste characteristic and organoleptic quality, we hope to find patterns among geno- types associates with differences in final fruit quality $[5,8]$.

\section{Materials and Methods}

\subsection{Instruments}

Polytrom PT3100 homogenizer (Kinematica AG, Switzerland) and an Eppendorf 5810R centrifuge (Eppendorf Iberica, Madrid, Spain) were used for sample treatment. Analysis was made using an Alliance liquid chromatographic system (Waters, Barcelona, Spain) equipped with a 2695 separation module, coupled to a 2996 photodiode array detector and a ZQ2000 mass detector. A thermostat column oven, a reverse-phase column $\mathrm{C}_{18}$ Tracer Excel $5 \mu \mathrm{m} 120$ OSDB $(250 \mathrm{~mm} \times 4.6 \mathrm{~mm})$ (Teknokroma, Barcelona, Spain), a ICSep ICE-COREGEL 87H3 column (Transgenomic), a ICSep ICE-COREGEL $87 \mathrm{H}$ guard kit, and an automatic injector were used for chromatographic separation. Empower 2 software was used for data acquisition. Sample temperature was $5^{\circ} \mathrm{C}$, column temperature was $25^{\circ} \mathrm{C}$ or $35^{\circ} \mathrm{C}$, and the UV-Vis spectra were recorded from 280 to $400 \mathrm{~nm}$. An HPLC system equipped with a Waters 515 HPLC pump, a Waters 2414 refractive index detector, a column Tracer Carbohydr $5 \mu \mathrm{m}(250 \mathrm{~mm} \times 4.5 \mathrm{~mm})$ (Teknokroma, Barcelona, Spain), and a $20 \mu \mathrm{L}$ loop Rheodyne injector were used for sugar analysis. Empower 2 software (Waters, Spain) was used for data processing.

\subsection{Plant Material and Sampling}

After full fruit development and at different stages of fruit maturity, representative samples were taken from healthy adult trees of the Field Collection of Citrus Germoplasm Bank held at Instituto Valenciano de Investigaciones Agrarias (IVIA) located at Moncada (Valencia, Spain). The cultivars studied were mandarins (clementine, satsume and others), hybrids, sweet oranges, grapefruits, pummelos, citrons, limes and lemons, and, where possible, we chose two different rootstocks for each cultivar (see Table 1). All cultivars shared the same environmental, cultural and soil conditions, thus the differences among cultivars were not influenced by climatic factors or crop techniques. Harvest was performed at eleven succesive periods during the 2009-2010 season between September and March (Table 1). Twenty fruits per cultivar were collected and separated into three replicates: 15 fruits per replicate were analysed for its content in total vitamin $\mathrm{C}$, organic acids and carbohydrates, and 5 fruits per replicate were peeled and used to obtain the juice using a Zumonat machine (Somatic-AMD, Spain) and analyzed for ${ }^{\circ}$ Brix with a refractometer (Atago Co. Ltd., Japan) and for acidity by titration with $0.1 \mathrm{~N} \mathrm{NaOH}$ 

at Different Stages of Ripening

Table 1. Name and harvest date of the citrus cultivars studied.

\begin{tabular}{|c|c|c|}
\hline Name & Genera species (Scientific name) & Rootstocks $^{1}$ \\
\hline \multicolumn{3}{|c|}{ Clementine mandarins } \\
\hline 1. "Fina" & C. clementina Hort. ex Tan. & Troyer \\
\hline 2. "Loretina" & C. clementina Hort. ex Tan. & Troyer and Cleopatra \\
\hline 3. “Arrufatina” & C. clementina Hort. ex Tan. & Cleopatra \\
\hline \multicolumn{3}{|c|}{ Satsume mandarins } \\
\hline 4. "Frost" & C. unshiu (Mak.) Marc. & Troyer and Cleopatra \\
\hline \multicolumn{3}{|c|}{ Other mandarins } \\
\hline 5. "Dancy" & C. tangerina Hort. ex Tan. & Carrizo \\
\hline 6. “Comun” & C. deliciosa Ten. & Carrizo \\
\hline \multicolumn{3}{|c|}{ Hybrids } \\
\hline 7. "Fortune" & C. clementina $\times$ C. tangerina ${ }^{2}$ & Troyer and Cleopatra \\
\hline 8. "Murcott" & C. reticulata $\times$ C. sinensis ${ }^{3}$ & Carrizo \\
\hline 9. “Ellendale" & C. reticulata $\times$ C. sinensis ${ }^{3}$ & Troyer \\
\hline \multicolumn{3}{|c|}{ Sweet Oranges } \\
\hline 10. “Navelate” & C. sinensis (L.) Osb. & Troyer and Cleopatra \\
\hline 11. "Valencia Late” & C. sinensis (L.) Osb. & Troyer and Cleopatra \\
\hline 12. “Sanguinelli” & C. sinensis (L.) Osb. & Troyer \\
\hline \multicolumn{3}{|c|}{ Grapefruits } \\
\hline 13. “Marsh” & C. paradisi Macf. & Troyer \\
\hline 14. “Star Ruby” & C. paradisi Macf. & Troyer \\
\hline \multicolumn{3}{|c|}{ Pummelos } \\
\hline 15. “Gil” & C. grandis (L.) Osb. & Troyer \\
\hline 16. “Deep Red” & C. grandis (L.) Osb. & Troyer \\
\hline \multicolumn{3}{|c|}{ Citrons } \\
\hline 17. “Arizona” & C. medica L. Var. ethrog Engl. & Troyer and Cleopatra \\
\hline \multicolumn{3}{|c|}{ Limes } \\
\hline 18. “Mejicana” & C. aurantifolia (Christm.) Swing & Troyer and Cleopatra \\
\hline 19. "Bearss" & C. latifolia Tan. & Troyer and Cleopatra \\
\hline \multicolumn{3}{|c|}{ Lemons } \\
\hline 20. "Fino" & C. limon (L.) Burm. f & Macrop and Sour \\
\hline Harvest Date & Number data & Cultivars sampled \\
\hline 07 September 2009 & Data I & 1 - 14, 16 - 20 \\
\hline 21 September 2009 & Data II & $1-20$ \\
\hline 05 October 2009 & Data III & $1-20$ \\
\hline 19 October 2009 & Data IV & $1-20$ \\
\hline 02 November 2009 & Data $\mathbf{V}$ & $1-20$ \\
\hline 16 November 2009 & Data VI & $1-20$ \\
\hline 30 November 2009 & Data VII & $1,4-17,19,20$ \\
\hline 14 December 2009 & Data VIII & $1,4-17,19,20$ \\
\hline 11 January 2010 & Data IX & $1,4-17,19,20$ \\
\hline 01 February 2010 & Data $\mathbf{X}$ & $5,7-16,20$ \\
\hline 22 March 2010 & Data XI & $7,8,10-16$ \\
\hline
\end{tabular}

${ }^{1}$ Troyer $=$ Troyer citrange $($ C. sinensis $($ L.) Osb. $\times$ Poncirus trifoliata $($ L.) Raf.); Cleopatra $=$ Cleopatra mandarin $($ C. reshni Hort. ex Tan.); Carrizo $=$ Carrizo citrange $($ C. sinensis $($ L. $)$ Osb. $\times$ Poncirus trifoliata (L.) Raf.); Macrop $=$ C. macrophylla Wester; Sour $=$ Sour orange $\left(\right.$ C. aurantium L.); ${ }^{2}$. reticulata Blanco $\times$ C. tangerina Hort. ex Tan.; $\left({ }^{3}\right)$ : C. reticulata Blanco $\times$ C. sinensis $($ L. $)$ Osb. 
using phenolphthalein as indicator.

\subsection{General Procedure for Extraction and Analysis of Vitamin C (Total Ascorbic Acid)}

Total vitamin C (ascorbic acid and dehydroascorbic acid) was determined by HPLC-DAD. The procedure used was the reduction of dehydroascorbic acid to ascorbic acid, using DL-dithiothreitol (DTT) as reducing reagent $[1,14]$. Briefly, $1 \mathrm{~mL}$ of filtered juice was mixed with $1 \mathrm{~mL}$ of $5 \%$ metaphosphoric acid solution, and then the sample was centrifuged at $4^{\circ} \mathrm{C}$ for $5 \mathrm{~min}$ at $10.000 \mathrm{rpm}$. One millilitre of supernatant was mixed with $200 \mu \mathrm{L}$ of DTT (20 $\mathrm{mg} / \mathrm{mL}$ ) and left to react for $2 \mathrm{~h}$ in the dark, then filtered through $0.45 \mu \mathrm{m}$ filter and used for total ascorbic acid determination by HPLC-DAD. A reverse-phase $\mathrm{C}_{18}$ column was used with an isocratic mobile phase of methanol: $0.6 \%$ acetic acid (5:95). The total run time was 10 $\min$ at $1 \mathrm{~mL} / \mathrm{min}$, and injection volume was $5 \mu \mathrm{L}$. The retention time of ascorbic acid was $3.8 \mathrm{~min}$, and quantification of ascorbic acid was performed at $245 \mathrm{~nm}$ by external standard calibration. L-Ascorbic acid and DTT were obtained from Sigma (Sigma Co., Barcelona, Spain) and Fluka (Sigma Co., Barcelona, Spain), respectively. All solvents used were of HPLC-grade and ultrapure water (Milli-Q) was used.

\subsection{General Procedure for Extraction and Analysis of Organic Acids}

$1 \mathrm{~mL}$ of filtered juice was mixed with $1 \mathrm{~mL}$ of $0.1 \%$ $\mathrm{H}_{2} \mathrm{SO}_{4}$ solution, and then the sample was centrifuged at $4^{\circ} \mathrm{C}$ for $5 \mathrm{~min}$ at $10.000 \mathrm{rpm}$. The supernatant were filtered through $0.45 \mu \mathrm{m}$ filter and analysed by HPLCDAD, and confirmed by HPLC-MS working in electrospray ion negative conditions [14]. The capillary voltage was $3.0 \mathrm{kV}$, cone voltage $23 \mathrm{~V}$, source temperature $100^{\circ} \mathrm{C}$, desolvation temperature $200^{\circ} \mathrm{C}$ and desolvation gas flow $400 \mathrm{~L} / \mathrm{Hr}$. Full data acquisition was performed scanning 100 to 400 uma in centroid mode. An ICSep ICE-COREGEL $87 \mathrm{H} 3$ column was used with an isocratic mobile phase of $0.1 \% \mathrm{H}_{2} \mathrm{SO}_{4}$ solution. The total run time was $20 \mathrm{~min}$ at $0.6 \mathrm{~mL} / \mathrm{min}$, and injection volume was 5 $\mu \mathrm{L}$. Compounds were indentified on the basis of comparing their retention times, UV-Vis spectra and mass spectrum data with corresponding authentic standards. Concentrations were determined using an external calibration curve with citric acid $\left(\mathrm{r}_{\mathrm{T}}=8.01 \mathrm{~min} ;[\mathrm{M}-\mathrm{H}]^{+} 191\right.$ $\mathrm{m} / \mathrm{z})$, malic acid $\left(\mathrm{r}_{\mathrm{T}}=9.41 \mathrm{~min} ;[\mathrm{M}-\mathrm{H}]^{+} 133 \mathrm{~m} / \mathrm{z}\right)$, and succinic acid $\left(\mathrm{r}_{\mathrm{T}}=11.43 \mathrm{~min} ;[\mathrm{M}-\mathrm{H}]^{+} 117 \mathrm{~m} / \mathrm{z}\right)$. All solvents were of HPLC-grade and ultrapure water (Milli-Q) was used. Standards were obtained from Sigma (Sigma Co., Barcelona, Spain).

\subsection{General Procedure for Extraction and Analysis of Carbohydrates}

$2 \mathrm{~mL}$ of juice were centrifuged at $10.000 \mathrm{rpm}$ for $5 \mathrm{~min}$ at $4{ }^{\circ} \mathrm{C}$. The samples were filtered through $0.45 \mu \mathrm{m}$ nylon filter and analyzed by HPLC using a column Tracer Carbohydr $250 \mathrm{~mm} \times 4.5 \mathrm{~mm}, 5 \mu \mathrm{m}$ (Teknokroma, Barcelona, Spain) and a mobile phase composed by acetonitrile:water (75:25) at a flow rate of $1 \mathrm{~mL} \cdot \mathrm{min}^{-1}$. Fructose, glucose and sucrose sugars were indentified comparing their retention time with a standard and quantified using an external calibration curve [14].

\subsection{Data Analysis}

Data were expressed as means. One-way ANOVA analyses were carried out with the Statgraphics Plus package, and the Duncan test method $(p<0.05)$ was applied to experimental data and to estimate significant differences amongst data.

\section{Results and Discussion}

The purpuse of the present study was the fast and efficient analysis of vitamin $\mathrm{C}$, organic acids and sugars using high-performance liquid chromatographic methods in twenty citrus cultivars and examine the influence of the variety and the different stages of fruit maturity. Also, where possible, we chose cultivars grafted onto different rootstocks. Because fruit weight, size, acidity, maturity index (a relationship between ${ }^{\circ}$ Brix and acidity), harvest time, chemical and nutritional composition are important quality traits, all of the citrus samples studied in this paper were harvested in the same field and year and also produced under the same conditions of climate to reduce additional sources of variance. After full fruit development and at different stages of fruit maturity, harvest was performed at eleven succesive periods except in those cultivars whose fruit was loss before (Table 1). Tables 2 and $\mathbf{3}$ sumarize the changes during maturation at dates $\mathbf{I}$ to $\mathbf{V}$, while in Tables 4-6 we report the changes during maturation at dates $\mathbf{V}$ to $\mathbf{X I}$.

In attempt to analyse the Citrus classification using the data obtained, we studied the vitamin C: citric acid: sucrose ratio comparing the influence of variety at commercial harvest stage (see Figure 1). We have chosen the best period time for harvest to carry out the study the chemical variability between varieties, noting that there are cultivars with a wide period of maintaining their fruit quality in the tree (fruit size, internal quality, good rind colour, taste and organoleptic properties).

${ }^{\circ}$ Brix, acidity and maturity index indicate the maturity status of the fruit. Obviously, the cultivars showed increasing maturity index values between September 7, 

at Different Stages of Ripening

Table 2. Changes in the maturity index and vitamin $C$ for different cultivars $(2,4,7,10,11,17$ - 20) and rootstocks (from I to V)

\begin{tabular}{|c|c|c|c|c|c|}
\hline \multirow{2}{*}{ Cultivar } & \multirow{2}{*}{ Date } & \multicolumn{2}{|c|}{ Maturity Index } & \multicolumn{2}{|c|}{ Vitamin $C^{1}$} \\
\hline & & Troyer & Cleopatra & Troyer & Cleopatra \\
\hline 2. "Loretina" & I & $5.59 \pm 0.92$ & $4.25 \pm 0.27$ & $53.41 \pm 3.21^{* *}$ & $61.81 \pm 5.17^{* *}$ \\
\hline 2. "Loretina" & II & $7.51 \pm 0.61$ & $8.27 \pm 0.11$ & $53.18 \pm 1.64$ & $58.35 \pm 3.06$ \\
\hline 2. "Loretina" & III & $9.15 \pm 1.92$ & $8.65 \pm 1.53$ & $54.08 \pm 1.01$ & $51.76 \pm 9.33$ \\
\hline 2. "Loretina" & IV & $11.26 \pm 0.61$ & $10.11 \pm 0.24$ & $47.10 \pm 3.63$ & $51.58 \pm 0.66$ \\
\hline 2. "Loretina" & $\mathbf{V}$ & $13.31 \pm 0.25$ & $12.39 \pm 0.53$ & $44.99 \pm 4.03^{* *}$ & $60.66 \pm 4.17^{* *}$ \\
\hline 4. "Frost" & I & $3.15 \pm 0.26$ & $2.91 \pm 0.31$ & $23.74 \pm 1.48$ & $22.77 \pm 1.57$ \\
\hline 4. "Frost" & II & $4.40 \pm 0.19$ & $3.91 \pm 0.20$ & $23.52 \pm 1.75$ & $22.69 \pm 1.88$ \\
\hline 4. "Frost" & III & $6.63 \pm 0.43^{*}$ & $4.74 \pm 0.91^{*}$ & $20.46 \pm 0.38$ & $21.22 \pm 1.73$ \\
\hline 4. "Frost" & IV & $7.64 \pm 0.34$ & $7.34 \pm 0.35$ & $25.57 \pm 0.67$ & $24.53 \pm 1.84$ \\
\hline 4. "Frost" & $\mathbf{V}$ & $10.41 \pm 0.25^{*}$ & $7.75 \pm 0.51^{*}$ & $26.56 \pm 0.99$ & $25.33 \pm 0.57$ \\
\hline 7. "Fortune" & I & $1.53 \pm 0.06$ & $1.55 \pm 0.03$ & $27.34 \pm 1.36^{*}$ & $23.30 \pm 2.83^{*}$ \\
\hline 7. "Fortune" & II & $1.76 \pm 0.05$ & $1.76 \pm 0.01$ & $20.98 \pm 0.72$ & $19.31 \pm 0.90$ \\
\hline 7. "Fortune" & III & $1.88 \pm 0.12$ & $1.76 \pm 0.05$ & $17.38 \pm 4.06$ & $18.67 \pm 1.87$ \\
\hline 7. "Fortune" & IV & $2.35 \pm 0.11$ & $2.45 \pm 0.08$ & $18.50 \pm 0.89$ & $15.90 \pm 0.17$ \\
\hline 7. “Fortune" & $\mathbf{v}$ & $2.89 \pm 0.16$ & $2.91 \pm 0.17$ & $16.35 \pm 0.98$ & $17.54 \pm 3.72$ \\
\hline 10. "Navelate" & $\mathbf{I}$ & $3.05 \pm 0.12$ & $2.67 \pm 0.33$ & $71.47 \pm 2.64^{* *}$ & $88.54 \pm 6.93^{* *}$ \\
\hline 10. “Navelate” & II & $3.68 \pm 0.06$ & $3.31 \pm 0.32$ & $65.18 \pm 3.87$ & $67.25 \pm 5.87$ \\
\hline 10. "Navelate” & III & $3.79 \pm 0.27$ & $3.58 \pm 0.43$ & $58.14 \pm 2.30$ & $61.63 \pm 3.16$ \\
\hline 10. "Navelate" & IV & $4.35 \pm 0.53$ & $4.02 \pm 0.62$ & $57.38 \pm 3.67$ & $61.47 \pm 3.03$ \\
\hline 10. "Navelate" & $\mathbf{V}$ & $6.04 \pm 0.28^{*}$ & $4.92 \pm 0.76^{*}$ & $53.39 \pm 1.65$ & $52.88 \pm 0.56$ \\
\hline 11. "Valencia L.” & I & $2.49 \pm 0.13$ & $2.32 \pm 0.13$ & $88.08 \pm 8.91$ & $83.52 \pm 0.75$ \\
\hline 11. "Valencia L.” & II & $2.65 \pm 0.26$ & $2.41 \pm 0.06$ & $74.86 \pm 2.08$ & $70.53 \pm 4.16$ \\
\hline 11. “Valencia L.” & III & $2.70 \pm 0.12$ & $2.54 \pm 0.23$ & $67.44 \pm 1.82$ & $68.05 \pm 2.39$ \\
\hline 11. "Valencia L.” & IV & $2.78 \pm 0.06$ & $2.46 \pm 0.19$ & $67.89 \pm 2.98$ & $66.74 \pm 2.98$ \\
\hline 11. "Valencia L.” & $\mathbf{V}$ & $2.99 \pm 0.30^{*}$ & $2.54 \pm 0.16^{*}$ & $58.10 \pm 2.72$ & $62.38 \pm 2.73$ \\
\hline 17. “Arizona” & I & $1.42 \pm 0.03^{*}$ & $1.29 \pm 0.06^{*}$ & $51.62 \pm 4.67^{* *}$ & $36.18 \pm 0.26^{* *}$ \\
\hline 17. “Arizona” & II & $1.41 \pm 0.02$ & $1.40 \pm 0.00$ & $43.87 \pm 0.05^{* *}$ & $31.47 \pm 0.25^{* *}$ \\
\hline 17. “Arizona” & III & $1.15 \pm 0.05^{*}$ & $1.29 \pm 0.05^{*}$ & $43.41 \pm 2.98^{* *}$ & $38.00 \pm 2.24^{* *}$ \\
\hline 17. “Arizona” & IV & $1.14 \pm 0.02$ & $1.17 \pm 0.02$ & $41.57 \pm 1.06^{* *}$ & $27.81 \pm 0.46^{* *}$ \\
\hline 17. “Arizona” & $\mathbf{v}$ & $1.17 \pm 0.03^{*}$ & $1.10 \pm 0.04^{*}$ & $38.94 \pm 4.59^{* *}$ & $27.94 \pm 0.98^{* *}$ \\
\hline 18. "Mejicana" & $\mathbf{I}$ & $1.35 \pm 0.01^{*}$ & $1.43 \pm 0.08^{*}$ & $52.24 \pm 2.43^{* *}$ & $43.76 \pm 1.18^{* *}$ \\
\hline 18. "Mejicana” & II & $1.31 \pm 0.01$ & $1.35 \pm 0.02$ & $45.02 \pm 1.76$ & $43.70 \pm 1.57$ \\
\hline 18. "Mejicana" & III & $1.19 \pm 0.05^{*}$ & $1.10 \pm 0.05^{*}$ & $36.53 \pm 3.00$ & $35.62 \pm 2.20$ \\
\hline 18. “Mejicana” & IV & $1.17 \pm 0.03$ & $1.22 \pm 0.04$ & $34.66 \pm 1.96$ & $32.46 \pm 1.11$ \\
\hline 18. "Mejicana" & $\mathbf{V}$ & $1.10 \pm 0.03$ & $1.10 \pm 0.02$ & $28.19 \pm 2.21$ & $30.58 \pm 1.22$ \\
\hline 19. “Bears” & I & $1.67 \pm 0.13$ & $1.61 \pm 0.06$ & $41.17 \pm 1.20$ & $43.10 \pm 2.22$ \\
\hline 19. "Bears" & II & $1.79 \pm 0.02$ & $1.66 \pm 0.10$ & $36.82 \pm 0.22$ & $34.50 \pm 1.69$ \\
\hline 19. "Bears" & III & $1.62 \pm 0.08^{*}$ & $1.46 \pm 0.10^{*}$ & $28.82 \pm 3.30^{* *}$ & $32.75 \pm 0.85^{* *}$ \\
\hline 19. "Bears" & IV & $1.60 \pm 0.16^{*}$ & $1.36 \pm 0.03^{*}$ & $30.91 \pm 0.88$ & $33.42 \pm 0.93$ \\
\hline \multirow[t]{2}{*}{ 19. "Bears" } & $\mathbf{V}$ & $1.53 \pm 0.05$ & $1.40 \pm 0.153$ & $29.39 \pm 2.56$ & $30.18 \pm 2.88$ \\
\hline & & Macrophylla & Sour orange & Macrophylla & Sour orange \\
\hline 20. "Fino" & $\mathbf{I}$ & $1.53 \pm 0.03^{*}$ & $1.43 \pm 0.03^{*}$ & $93.15 \pm 3.76^{* *}$ & $73.94 \pm 0.32^{* *}$ \\
\hline 20. "Fino" & II & $1.38 \pm 0.02$ & $1.37 \pm 0.01$ & $85.79 \pm 2.24^{* *}$ & $70.29 \pm 0.29^{* *}$ \\
\hline 20. "Fino" & III & $1.33 \pm 0.04$ & $1.30 \pm 0.06$ & $76.30 \pm 3.17^{* *}$ & $63.97 \pm 2.35^{* *}$ \\
\hline 20. “Fino" & IV & $1.27 \pm 0.08$ & $1.30 \pm 0.07$ & $69.95 \pm 2.62^{* *}$ & $63.89 \pm 5.29^{* *}$ \\
\hline 20. "Fino" & $\mathbf{v}$ & $1.27 \pm 0.06$ & $1.26 \pm 0.04$ & $65.18 \pm 1.56^{* *}$ & $52.66 \pm 2.12^{* *}$ \\
\hline
\end{tabular}

${ }^{\mathrm{x}}$ Data are expressed as mean $(\mathrm{n}=6) \pm$ standard deviation; ${ }^{1} \mathrm{mg}$ vitamin $\mathrm{C}$ total/100mL juice; ${ }^{* * * *}$ Cultivars with significantly different values of maturity index or vitamin $\mathrm{C}$ between rootstocks (same date collection). 
Table 3. Maturity index and vitamin $\mathrm{C}$ for different cultivars during maturation (from $\mathrm{I}$ to $\mathrm{V})^{\mathrm{X}}$.

\begin{tabular}{|c|c|c|c|}
\hline Cultivar $^{1}$ & Date & Maturity Index & ${\text { Vitamin } C^{2}}^{2}$ \\
\hline 1. “Fina” & I & $2.55 \pm 0.28$ & $62.02 \pm 1.92$ \\
\hline 1. “Fina" & II & $3.86 \pm 0.23$ & $50.64 \pm 0.83$ \\
\hline 1. “Fina" & III & $5.64 \pm 0.74$ & $49.76 \pm 0.70$ \\
\hline 1. "Fina" & IV & $8.34 \pm 0.59$ & $50.30 \pm 2.84$ \\
\hline 1. “Fina” & $\mathbf{V}$ & $11.78 \pm 0.74$ & $48.10 \pm 4.22$ \\
\hline 3. "Arrufatina" & I & $4.32 \pm 0.85$ & $62.96 \pm 4.54$ \\
\hline 3. "Arrufatina” & II & $8.07 \pm 0.27$ & $55.90 \pm 6.66$ \\
\hline 3. "Arrufatina” & III & $10.65 \pm 0.18$ & $56.32 \pm 1.43$ \\
\hline 3. "Arrufatina” & IV & $11.57 \pm 0.65$ & $60.74 \pm 0.62$ \\
\hline 3. “Arrufatina” & $\mathbf{V}$ & $14.13 \pm 0.54$ & $54.80 \pm 1.39$ \\
\hline 5. “Dancy” & $\mathbf{I}$ & $1.36 \pm 0.09$ & $28.51 \pm 1.39$ \\
\hline 5. "Dancy" & II & $1.56 \pm 0.03$ & $27.73 \pm 3.92$ \\
\hline 5. “Dancy" & III & $1.99 \pm 0.13$ & $24.80 \pm 1.00$ \\
\hline 5. “Dancy" & IV & $2.70 \pm 0.20$ & $26.51 \pm 0.87$ \\
\hline 5. “Dancy" & $\mathbf{V}$ & $3.61 \pm 0.08$ & $28.03 \pm 0.58$ \\
\hline 6. "Comun" & $\mathbf{I}$ & $1.43 \pm 0.05$ & $36.58 \pm 1.34$ \\
\hline 6. "Comun" & II & $1.49 \pm 0.02$ & $36.11 \pm 3.72$ \\
\hline 6. "Comun" & III & $1.61 \pm 0.06$ & $29.58 \pm 0.93$ \\
\hline 6. “Comun” & IV & $1.87 \pm 0.06$ & $32.45 \pm 0.85$ \\
\hline 6. “Comun” & $\mathbf{V}$ & $2.33 \pm 0.06$ & $31.62 \pm 1.52$ \\
\hline 8. “Murcott" & $\mathbf{I}$ & $1.81 \pm 0.11$ & $20.03 \pm 0.87$ \\
\hline 8. “Murcott” & II & $2.20 \pm 0.15$ & $18.80 \pm 0.50$ \\
\hline 8. “Murcott" & III & $2.57 \pm 0.06$ & $20.18 \pm 1.25$ \\
\hline 8. “Murcott" & IV & $3.05 \pm 0.08$ & $20.34 \pm 0.47$ \\
\hline 8. “Murcott" & $\mathbf{V}$ & $3.88 \pm 0.16$ & $19.98 \pm 1.50$ \\
\hline 9. "Ellendale" & $\mathbf{I}$ & $1.66 \pm 0.14$ & $63.70 \pm 3.16$ \\
\hline 9. “Ellendale" & II & $1.68 \pm 0.07$ & $57.92 \pm 2.13$ \\
\hline 9. "Ellendale" & III & $1.75 \pm 0.05$ & $51.55 \pm 1.12$ \\
\hline 9. "Ellendale" & IV & $2.34 \pm 0.34$ & $47.98 \pm 1.86$ \\
\hline 9. “Ellendale” & $\mathbf{V}$ & $2.69 \pm 0.15$ & $55.94 \pm 6.75$ \\
\hline 12. "Sanguinelli" & $\mathbf{I}$ & $2.98 \pm 0.10$ & $70.58 \pm 4.29$ \\
\hline 12. "Sanguinelli" & II & $3.16 \pm 0.20$ & $64.43 \pm 2.22$ \\
\hline 12. "Sanguinelli" & III & $3.21 \pm 0.51$ & $58.37 \pm 8.57$ \\
\hline 12. "Sanguinelli" & IV & $3.80 \pm 0.24$ & $60.78 \pm 0.85$ \\
\hline 12. "Sanguinelli" & $\mathbf{V}$ & $4.41 \pm 0.39$ & $57.22 \pm 1.82$ \\
\hline 13. “Marsh” & $\mathbf{I}$ & $4.08 \pm 0.13$ & $84.14 \pm 2.69$ \\
\hline 13. "Marsh" & II & $4.21 \pm 0.27$ & $63.09 \pm 3.06$ \\
\hline 13. "Marsh" & III & $4.49 \pm 0.50$ & $49.41 \pm 2.06$ \\
\hline 13. "Marsh" & IV & $4.56 \pm 0.28$ & $48.27 \pm 1.48$ \\
\hline 13. “Marsh” & $\mathbf{V}$ & $4.52 \pm 0.09$ & $42.86 \pm 2.12$ \\
\hline 14. "Star Ruby" & I & $3.40 \pm 0.20$ & $104.50 \pm 6.29$ \\
\hline 14. "Star Ruby" & II & $3.65 \pm 0.21$ & $71.89 \pm 12.37$ \\
\hline 14. "Star Ruby" & III & $4.01 \pm 0.11$ & $59.28 \pm 7.02$ \\
\hline 14. "Star Ruby" & IV & $4.29 \pm 0.12$ & $49.54 \pm 4.34$ \\
\hline 14. "Star Ruby" & $\mathbf{V}$ & $4.30 \pm 0.10$ & $53.14 \pm 2.02$ \\
\hline 15. “Gil” & II & $6.55 \pm 0.28$ & $67.98 \pm 4.52$ \\
\hline 15. “Gil” & III & $5.74 \pm 0.89$ & $58.58 \pm 2.93$ \\
\hline 15. “Gil” & IV & $4.17 \pm 0.25$ & $47.34 \pm 4.98$ \\
\hline 15. “Gil” & $\mathbf{V}$ & $3.94 \pm 0.10$ & $49.90 \pm 1.85$ \\
\hline 16. “Deep Red” & I & $7.80 \pm 0.46$ & $110.88 \pm 4.80$ \\
\hline 16. "Deep Red" & II & $7.66 \pm 0.52$ & $90.22 \pm 0.61$ \\
\hline 16. “Deep Red” & III & $6.76 \pm 0.46$ & $68.53 \pm 3.92$ \\
\hline 16. “Deep Red” & IV & $6.74 \pm 0.39$ & $54.45 \pm 2.28$ \\
\hline 16. "Deep Red" & $\mathbf{V}$ & $7.17 \pm 0.77$ & $56.32 \pm 3.74$ \\
\hline
\end{tabular}

${ }^{\mathrm{x}}$ Data are expressed as mean $(\mathrm{n}=6) \pm$ standard deviation; ${ }^{1}$ Rootstocks: Troyer for 1, 7, 9, 12 - 16; Carrizo for 5, 6, 8; Cleopatra for 3. ${ }^{2} \mathrm{mg}$ vitamin $\mathrm{C}$ total $/ 100 \mathrm{~mL}$ juice. 
Table 4. Changes in the maturity index, vitamin $\mathrm{C}$, organic acids and sugars for mandarins, hybrids and oranges during maturation (from V to XI) ${ }^{\mathrm{X}}$.

\begin{tabular}{|c|c|c|c|c|c|c|c|c|c|}
\hline \multirow{2}{*}{ Cultivar $^{1}$} & \multirow{2}{*}{ Date } & \multirow{2}{*}{ Mat. Index } & \multirow{2}{*}{ Vitamin $C^{2}$} & \multicolumn{3}{|c|}{ Organic acids (g/L juice) } & \multicolumn{3}{|c|}{ Sugars (g/L juice) } \\
\hline & & & & Citric & Malic & Succinic & Fructose & Glucose & Sucrose \\
\hline 1. “Fina” & $\mathbf{V}$ & $11.78 \pm 0.74$ & $48.10 \pm 4.22$ & $8.33 \pm 1.04$ & $7.62 \pm 0.09$ & $0.54 \pm 0.02$ & $11.58 \pm 2.59$ & $9.50 \pm 1.56$ & $45.09 \pm 7.64$ \\
\hline 1. "Fina" & VI & $13.51 \pm 0.92$ & $46.54 \pm 0.82$ & $7.41 \pm 0.21$ & $8.18 \pm 0.17$ & $0.78 \pm 0.14$ & $11.49 \pm 0.07$ & $9.56 \pm 0.50$ & $57.92 \pm 0.75$ \\
\hline 1. “Fina” & VII & $13.61 \pm 1.38$ & $49.36 \pm 1.91$ & $6.86 \pm 0.22$ & $9.66 \pm 0.21$ & $0.68 \pm 0.05$ & $17.58 \pm 1.05$ & $15.05 \pm 0.38$ & $78.40 \pm 3.63$ \\
\hline 1. "Fina" & VIII & $15.39 \pm 0.29$ & $45.89 \pm 0.92$ & $5.76 \pm 0.29$ & $9.54 \pm 0.38$ & $0.61 \pm 0.08$ & $18.36 \pm 1.80$ & $14.05 \pm 0.93$ & $48.31 \pm 3.35$ \\
\hline 1. "Fina" & IX & $15.55 \pm 0.39$ & $48.83 \pm 0.78$ & $9.34 \pm 0.32$ & $10.76 \pm 0.26$ & $0.64 \pm 0.00$ & $19.30 \pm 1.49$ & $15.87 \pm 3.14$ & $78.77 \pm 7.41$ \\
\hline 2. "Loretina" & $\mathbf{V}$ & $13.31 \pm 0.25$ & $44.99 \pm 4.03$ & $7.75 \pm 0.20$ & $6.59 \pm 0.05$ & $0.61 \pm 0.03$ & $13.26 \pm 0.61$ & $12.57 \pm 0.92$ & $52.45 \pm 2.60$ \\
\hline 2. “Loretina” & VI & $13.40 \pm 0.19$ & $54.21 \pm 5.14$ & $8.13 \pm 0.38$ & $8.08 \pm 0.41$ & $0.83 \pm 0.06$ & $15.62 \pm 1.93$ & $13.90 \pm 1.49$ & $53.31 \pm 3.30$ \\
\hline 3. "Arrufatina” & $\mathbf{V}$ & $14.13 \pm 0.54$ & $54.80 \pm 1.39$ & $9.51 \pm 1.09$ & $3.18 \pm 0.41$ & $1.50 \pm 0.05$ & $9.92 \pm 0.64$ & $9.78 \pm 0.38$ & $61.42 \pm 2.87$ \\
\hline 3. “Arrufatina" & VI & $14.46 \pm 1.02$ & $62.35 \pm 5.58$ & $8.60 \pm 0.19$ & $9.43 \pm 0.17$ & $1.50 \pm 0.05$ & $11.05 \pm 1.15$ & $11.54 \pm 1.31$ & $56.62 \pm 2.53$ \\
\hline 4. "Frost" & $\mathbf{V}$ & $10.41 \pm 0.25$ & $26.56 \pm 0.992$ & $9.51 \pm 1.09$ & $3.18 \pm 0.41$ & $1.50 \pm 0.05$ & $11.24 \pm 0.94$ & $11.33 \pm 0.53$ & $38.84 \pm 0.51$ \\
\hline 4. "Frost" & VI & $10.74 \pm 0.42$ & $28.37 \pm 0.55$ & $9.82 \pm 0.26$ & $3.79 \pm 0.28$ & $1.58 \pm 0.01$ & $14.71 \pm 0.65$ & $14.51 \pm 0.68$ & $49.90 \pm 3.77$ \\
\hline 4. "Frost" & VII & $13.65 \pm 0.19$ & $30.43 \pm 0.75$ & $10.17 \pm 0.06$ & $4.57 \pm 0.03$ & $1.30 \pm 0.03$ & $20.97 \pm 0.48$ & $18.37 \pm 0.74$ & $67.35 \pm 1.09$ \\
\hline 4. "Frost" & VIII & $14.22 \pm 0.21$ & $20.72 \pm 0.84$ & $10.06 \pm 0.78$ & $4.66 \pm 0.46$ & $1.26 \pm 0.24$ & $17.63 \pm 0.51$ & $14.26 \pm 0.87$ & $44.48 \pm 6.21$ \\
\hline 4. "Frost" & IX & $15.43 \pm 0.71$ & $26.06 \pm 1.48$ & $10.17 \pm 0.58$ & $4.63 \pm 0.37$ & $1.35 \pm 0.04$ & $14.68 \pm 1.71$ & $12.75 \pm 1.65$ & $52.60 \pm 2.78$ \\
\hline 5. "Dancy" & $\mathbf{V}$ & $3.61 \pm 0.08$ & $28.03 \pm 0.58$ & $25.70 \pm 1.21$ & $3.07 \pm 0.16$ & $1.60 \pm 0.07$ & $10.65 \pm 0.50$ & $10.52 \pm 0.41$ & $35.28 \pm 1.91$ \\
\hline 5. "Dancy" & VI & $5.06 \pm 0.32$ & $32.85 \pm 1.63$ & $20.75 \pm 0.05$ & $4.05 \pm 0.18$ & $1.75 \pm 0.02$ & $15.13 \pm 1.21$ & $15.42 \pm 0.57$ & $41.43 \pm 1.12$ \\
\hline 5. "Dancy" & VII & $5.74 \pm 0.19$ & $33.18 \pm 2.10$ & $20.72 \pm 0.17$ & $5.19 \pm 0.11$ & $1.37 \pm 0.01$ & $24.48 \pm 1.18$ & $22.90 \pm 1.83$ & $75.38 \pm 4.68$ \\
\hline 5. "Dancy" & VIII & $7.62 \pm 0.82$ & $23.25 \pm 2.16$ & $14.43 \pm 1.64$ & $5.33 \pm 0.19$ & $1.27 \pm 0.13$ & $12.19 \pm 1.17$ & $10.23 \pm 1.28$ & $46.55 \pm 2.39$ \\
\hline 5. "Dancy" & IX & $8.33 \pm 0.53$ & $25.66 \pm 0.99$ & $12.43 \pm 0.29$ & $4.89 \pm 0.15$ & $1.04 \pm 0.02$ & $13.97 \pm 1.12$ & $13.24 \pm 0.66$ & $50.20 \pm 3.46$ \\
\hline 5. "Dancy" & $\mathbf{X}$ & $10.25 \pm 0.27$ & $27.90 \pm 1.09$ & $14.27 \pm 0.38$ & $5.46 \pm 0.15$ & $1.08 \pm 0.02$ & $12.55 \pm 0.50$ & $10.29 \pm 0.19$ & $57.19 \pm 1.63$ \\
\hline 6. “Comun” & $\mathbf{V}$ & $2.33 \pm 0.06$ & $31.62 \pm 1.52$ & $41.38 \pm 0.71$ & $3.24 \pm 0.19$ & $1.72 \pm 0.24$ & $6.84 \pm 0.38$ & $6.91 \pm 0.43$ & $24.60 \pm 1.77$ \\
\hline 6. "Comun" & VI & $2.83 \pm 0.12$ & $35.90 \pm 0.10$ & $39.73 \pm 0.40$ & $4.61 \pm 0.08$ & $1.81 \pm 0.12$ & $8.87 \pm 1.39$ & $8.50 \pm 1.30$ & $29.80 \pm 4.77$ \\
\hline 6. “Comun” & VII & $3.16 \pm 0.20$ & $38.61 \pm 1.64$ & $35.83 \pm 0.42$ & $6.08 \pm 0.21$ & $2.35 \pm 0.11$ & $9.64 \pm 0.22$ & $8.74 \pm 0.69$ & $35.32 \pm 0.93$ \\
\hline 6. “Comun” & VIII & $3.46 \pm 0.11$ & $30.34 \pm 1.44$ & $37.41 \pm 1.43$ & $6.39 \pm 0.68$ & $2.41 \pm 0.16$ & $9.69 \pm 0.89$ & $8.52 \pm 0.73$ & $38.78 \pm 1.54$ \\
\hline 6. “Comun” & IX & $5.74 \pm 0.15$ & $30.08 \pm 0.75$ & $26.08 \pm 0.23$ & $6.12 \pm 0.08$ & $1.32 \pm 0.02$ & $12.11 \pm 0.59$ & $11.92 \pm 0.85$ & $52.70 \pm 2.41$ \\
\hline 7. "Fortune" & $\mathbf{V}$ & $2.89 \pm 0.16$ & $16.35 \pm 0.98$ & $29.90 \pm 1.63$ & $3.09 \pm 0.18$ & $1.99 \pm 0.07$ & $8.74 \pm 0.35$ & $8.58 \pm 0.47$ & $30.61 \pm 1.13$ \\
\hline 7. "Fortune" & VI & $3.70 \pm 0.06$ & $18.56 \pm 0.75$ & $28.37 \pm 0.18$ & $4.31 \pm 0.23$ & $3.02 \pm 0.40$ & $11.03 \pm 0.43$ & $10.27 \pm 0.73$ & $37.45 \pm 2.19$ \\
\hline 7. "Fortune" & VII & $4.57 \pm 0.29$ & $19.30 \pm 0.98$ & $23.43 \pm 0.50$ & $4.58 \pm 0.37$ & $2.91 \pm 0.08$ & $13.00 \pm 0.57$ & $12.44 \pm 0.56$ & $39.83 \pm 1.98$ \\
\hline 7. "Fortune" & VIII & $4.98 \pm 0.27$ & $13.70 \pm 1.02$ & $20.44 \pm 1.02$ & $4.83 \pm 0.73$ & $2.45 \pm 0.36$ & $12.14 \pm 0.33$ & $10.58 \pm 0.22$ & $39.74 \pm 1.63$ \\
\hline 7. "Fortune" & IX & $5.39 \pm 0.07$ & $15.17 \pm 0.60$ & $22.18 \pm 0.31$ & $5.36 \pm 0.07$ & $3.23 \pm 0.04$ & $14.42 \pm 0.60$ & $13.77 \pm 0.69$ & $50.16 \pm 1.96$ \\
\hline 7. "Fortune" & $\mathbf{x}$ & $7.06 \pm 0.28$ & $21.33 \pm 0.70$ & $20.19 \pm 0.11$ & $5.38 \pm 0.05$ & $2.92 \pm 0.06$ & $13.52 \pm 0.22$ & $12.10 \pm 0.14$ & $54.02 \pm 1.08$ \\
\hline 7. "Fortune" & XI & $8.62 \pm 0.06$ & $22.21 \pm 1.54$ & $20.87 \pm 0.58$ & $5.31 \pm 0.54$ & $2.41 \pm 0.28$ & $16.96 \pm 1.58$ & $15.51 \pm 1.58$ & $60.88 \pm 2.15$ \\
\hline 8. “Murcott” & $\mathbf{V}$ & $3.88 \pm 0.16$ & $19.98 \pm 1.50$ & $19.58 \pm 0.58$ & $3.05 \pm 0.14$ & $1.55 \pm 0.13$ & $8.71 \pm 0.18$ & $8.56 \pm 0.27$ & $29.05 \pm 0.29$ \\
\hline 8. “Murcott” & VI & $4.42 \pm 0.21$ & $21.41 \pm 1.59$ & $23.54 \pm 0.28$ & $3.80 \pm 0.12$ & $1.96 \pm 0.01$ & $12.71 \pm 2.71$ & $12.05 \pm 2.36$ & $32.74 \pm 1.48$ \\
\hline 8. “Murcott” & VII & $5.51 \pm 0.30$ & $20.88 \pm 0.38$ & $21.11 \pm 0.27$ & $3.78 \pm 0.16$ & $2.02 \pm 0.06$ & $13.83 \pm 0.56$ & $13.21 \pm 0.24$ & $41.54 \pm 2.85$ \\
\hline 8. “Murcott” & VIII & $6.16 \pm 0.10$ & $13.76 \pm 0.52$ & $19.72 \pm 0.75$ & $5.32 \pm 0.29$ & $1.90 \pm 0.13$ & $12.45 \pm 0.66$ & $10.32 \pm 0.62$ & $39.10 \pm 1.51$ \\
\hline 8. “Murcott” & IX & $7.64 \pm 0.07$ & $15.52 \pm 1.47$ & $19.84 \pm 0.10$ & $4.33 \pm 0.02$ & $1.18 \pm 0.02$ & $17.28 \pm 0.29$ & $15.77 \pm 0.40$ & $51.80 \pm 1.53$ \\
\hline 8. "Murcott” & $\mathbf{X}$ & $8.57 \pm 0.33$ & $14.66 \pm 0.93$ & $16.48 \pm 0.09$ & $4.77 \pm 0.02$ & $1.01 \pm 0.07$ & $15.60 \pm 0.73$ & $13.34 \pm 0.53$ & $49.85 \pm 2.74$ \\
\hline 8. “Murcott” & XI & $11.50 \pm 0.45$ & $14.51 \pm 0.35$ & $17.26 \pm 0.11$ & $3.66 \pm 0.04$ & $0.76 \pm 0.09$ & $21.74 \pm 0.50$ & $19.00 \pm 1.03$ & $60.52 \pm 1.80$ \\
\hline 9. "Ellendale" & $\mathbf{V}$ & $2.69 \pm 0.15$ & $55.94 \pm 6.75$ & $40.89 \pm 0.70$ & $6.69 \pm 0.69$ & $1.41 \pm 0.02$ & $9.09 \pm 0.42$ & $9.63 \pm 0.64$ & $30.63 \pm 1.82$ \\
\hline 9. “Ellendale” & VI & $2.86 \pm 0.27$ & $54.0 \pm 2.47$ & $42.23 \pm 1.82$ & $5.96 \pm 0.17$ & $1.89 \pm 0.13$ & $11.98 \pm 0.52$ & $11.67 \pm 0.49$ & $39.02 \pm 4.06$ \\
\hline
\end{tabular}




\section{Continued}

\begin{tabular}{|c|c|c|c|c|c|c|c|c|c|}
\hline 9. "Ellendale" & VII & $4.02 \pm 0.40$ & $50.61 \pm 1.45$ & $34.38 \pm 0.15$ & $6.29 \pm 0.25$ & $1.32 \pm 0.01$ & $13.08 \pm 0.78$ & $13.36 \pm 0.25$ & $39.46 \pm 1.18$ \\
\hline 9. "Ellendale" & VIII & $4.12 \pm 0.31$ & $40.46 \pm 4.94$ & $32.64 \pm 1.76$ & $8.04 \pm 0.27$ & $1.40 \pm 0.16$ & $18.57 \pm 0.98$ & $13.86 \pm 1.95$ & $62.64 \pm 3.55$ \\
\hline 9. "Ellendale" & IX & $5.77 \pm 0.11$ & $40.46 \pm 2.59$ & $26.08 \pm 0.48$ & $7.36 \pm 0.17$ & $1.00 \pm 0.03$ & $13.85 \pm 1.42$ & $5.72 \pm 0.80$ & $60.25 \pm 9.01$ \\
\hline 9. "Ellendale" & $\mathbf{X}$ & $7.32 \pm 0.21$ & $41.39 \pm 2.95$ & $20.89 \pm 0.15$ & $7.69 \pm 0.06$ & $0.95 \pm 0.04$ & $12.96 \pm 0.83$ & $1.48 \pm 1.25$ & $54.13 \pm 1.88$ \\
\hline 10. "Navelate" & $\mathbf{V}$ & $04 \pm 0.28$ & $53.39 \pm 1.65$ & $17.49 \pm 0.52$ & $6.15 \pm 0.44$ & $.39 \pm 0.04$ & $10.92 \pm 0.21$ & $81 \pm 0.33$ & 2.74 \\
\hline 10. "Navelate" & VI & $6.84 \pm 0.44$ & $55.68 \pm 0.82$ & $15.72 \pm 0.86$ & $6.56 \pm 0.13$ & $1.89 \pm 0.04$ & $11.18 \pm 1.29$ & $10.45 \pm 0.60$ & $35.53 \pm 1.86$ \\
\hline 10. “Navelate" & VII & $7.60 \pm 0.19$ & $54.50 \pm 0.44$ & $13.97 \pm 0.10$ & $7.85 \pm 0.08$ & $1.84 \pm 0.03$ & $19.12 \pm 2.25$ & $16.92 \pm 1.27$ & $44.80 \pm 1.75$ \\
\hline 10. "Navelate" & VIII & $7.89 \pm 0.99$ & $41.39 \pm 1.54$ & $14.41 \pm 0.61$ & $7.66 \pm 0.21$ & $1.65 \pm 0.38$ & $21.15 \pm 3.42$ & $14.96 \pm 0.99$ & $58.57 \pm 6.73$ \\
\hline 10. "Navelate" & IX & $10.39 \pm 0.16$ & $48.00 \pm 4.13$ & $13.63 \pm 0.24$ & $8.14 \pm 0.16$ & $1.26 \pm 0.01$ & $17.54 \pm 0.71$ & $16.45 \pm 0.31$ & $43.47 \pm 1.86$ \\
\hline 10. “Navelate” & $\mathbf{X}$ & $10.94 \pm 0.64$ & $38.22 \pm 1.36$ & $12.81 \pm 0.18$ & $6.99 \pm 0.04$ & $1.06 \pm 0.32$ & $14.77 \pm 0.29$ & $12.82 \pm 0.70$ & $41.18 \pm 0.99$ \\
\hline 10. “Navelate” & XI & $11.86 \pm 0.55$ & $48.03 \pm 5.29$ & $13.70 \pm 0.11$ & $7.42 \pm 0.04$ & $1.17 \pm 0.04$ & $18.12 \pm 0.60$ & $16.11 \pm 0.90$ & $43.22 \pm 0.79$ \\
\hline 11. “Valencia L.” & $\mathbf{V}$ & $2.99 \pm 0.30$ & $58.10 \pm 2.72$ & $31.31 \pm 3.52$ & $6.65 \pm 0.35$ & $1.29 \pm 0.03$ & $10.65 \pm 0.91$ & $10.20 \pm 0.11$ & $21.97 \pm 1.73$ \\
\hline 11. “Valencia L.” & VI & $3.46 \pm 0.17$ & $63.78 \pm 0.44$ & $29.86 \pm 0.48$ & $7.58 \pm 0.17$ & $1.86 \pm 0.05$ & $11.25 \pm 0.60$ & $10.87 \pm 0.54$ & $26.22 \pm 1.29$ \\
\hline 11. “Valencia L.” & VII & $3.53 \pm 0.21$ & $59.30 \pm 2.71$ & $29.20 \pm 0.34$ & $7.80 \pm 0.07$ & $1.32 \pm 0.08$ & $14.75 \pm 0.72$ & $15.18 \pm 1.95$ & $34.61 \pm 3.56$ \\
\hline 11. “Valencia L.” & VIII & $4.20 \pm 0.07$ & $43.04 \pm 1.41$ & $24.81 \pm 0.59$ & $7.97 \pm 0.31$ & $1.50 \pm 0.09$ & $20.29 \pm 3.66$ & $21.94 \pm 4.04$ & $60.27 \pm 8.08$ \\
\hline 11. “Valencia L.” & IX & $5.74 \pm 0.32$ & $52.11 \pm 3.17$ & $23.33 \pm 0.44$ & $8.64 \pm 0.26$ & $1.40 \pm 0.01$ & $16.41 \pm 0.23$ & $15.84 \pm 0.55$ & $35.36 \pm 0.63$ \\
\hline 11. “Valencia L.” & $\mathbf{X}$ & $5.99 \pm 0.01$ & $46.66 \pm 0.05$ & $21.41 \pm 0.53$ & $8.32 \pm 0.21$ & $1.51 \pm 0.06$ & $13.75 \pm 0.42$ & $12.60 \pm 0.61$ & $34.23 \pm 1.08$ \\
\hline 11. “Valencia L.” & XI & $6.38 \pm 0.34$ & $51.89 \pm 2.19$ & $19.33 \pm 0.54$ & $7.00 \pm 0.29$ & $1.18 \pm 0.02$ & $14.33 \pm 0.45$ & $13.70 \pm 0.33$ & $36.63 \pm 0.83$ \\
\hline 12. "Sanguinelli" & $\mathbf{V}$ & $4.41 \pm 0.39$ & $57.22 \pm 1.82$ & $26.78 \pm 0.38$ & $6.96 \pm 0.43$ & $0.74 \pm 0.06$ & $15.53 \pm 1.09$ & $15.95 \pm 1.01$ & $31.08 \pm 2.26$ \\
\hline 12. "Sanguinelli" & VI & $4.61 \pm 0.47$ & $59.28 \pm 0.77$ & $24.79 \pm 1.58$ & $7.38 \pm 0.50$ & $0.87 \pm 0.13$ & $16.57 \pm 0.43$ & $16.33 \pm 0.36$ & $30.31 \pm 1.00$ \\
\hline 12. "Sanguinelli" & VII & $5.14 \pm 0.12$ & $56.29 \pm 0.45$ & $24.07 \pm 0.39$ & $7.44 \pm 0.18$ & $0.54 \pm 0.03$ & $21.70 \pm 0.96$ & $20.78 \pm 1.11$ & $39.58 \pm 2.51$ \\
\hline 12. "Sanguinelli" & VIII & $5.17 \pm 0.19$ & $37.41 \pm 1.99$ & $20.57 \pm 0.37$ & $6.72 \pm 0.03$ & $0.52 \pm 0.03$ & $19.13 \pm 1.33$ & $14.18 \pm 1.30$ & $33.81 \pm 5.46$ \\
\hline 12. "Sanguinelli" & IX & $7.22 \pm 0.11$ & $46.62 \pm 0.33$ & $20.01 \pm 0.17$ & $7.28 \pm 0.13$ & $0.51 \pm 0.03$ & $19.05 \pm 0.58$ & $18.20 \pm 0.69$ & $35.82 \pm 0.50$ \\
\hline 12. "Sanguinelli" & $\mathbf{X}$ & $7.64 \pm 0.36$ & $40.56 \pm 1.81$ & $18.04 \pm 1.13$ & $6.74 \pm 0.48$ & $0.45 \pm 0.04$ & $16.47 \pm 0.43$ & $15.19 \pm 0.80$ & $34.79 \pm 0.78$ \\
\hline 12. "Sanguinelli" & XI & $8.44 \pm 0.43$ & $46.54 \pm 2.00$ & $16.94 \pm 2.06$ & $7.24 \pm 1.08$ & $0.43 \pm 0.19$ & $21.57 \pm 2.26$ & $20.21 \pm 2.59$ & $35.13 \pm 3.68$ \\
\hline
\end{tabular}

${ }^{x}$ Data are expressed as mean (maturity index and vitamin $C: n=6$; organic acids and sugars: $n=3$ ) \pm standard deviation; ${ }^{1}$ Rootstocks: Troyer for $\mathbf{1}, \mathbf{2 , 4}, 7, \mathbf{9}$ 12; Carrizo for 5, 6, 8; Cleopatra for $3 ;{ }^{2} \mathrm{mg}$ vitamin $\mathrm{C}$ total $/ 100 \mathrm{~mL}$ juice.

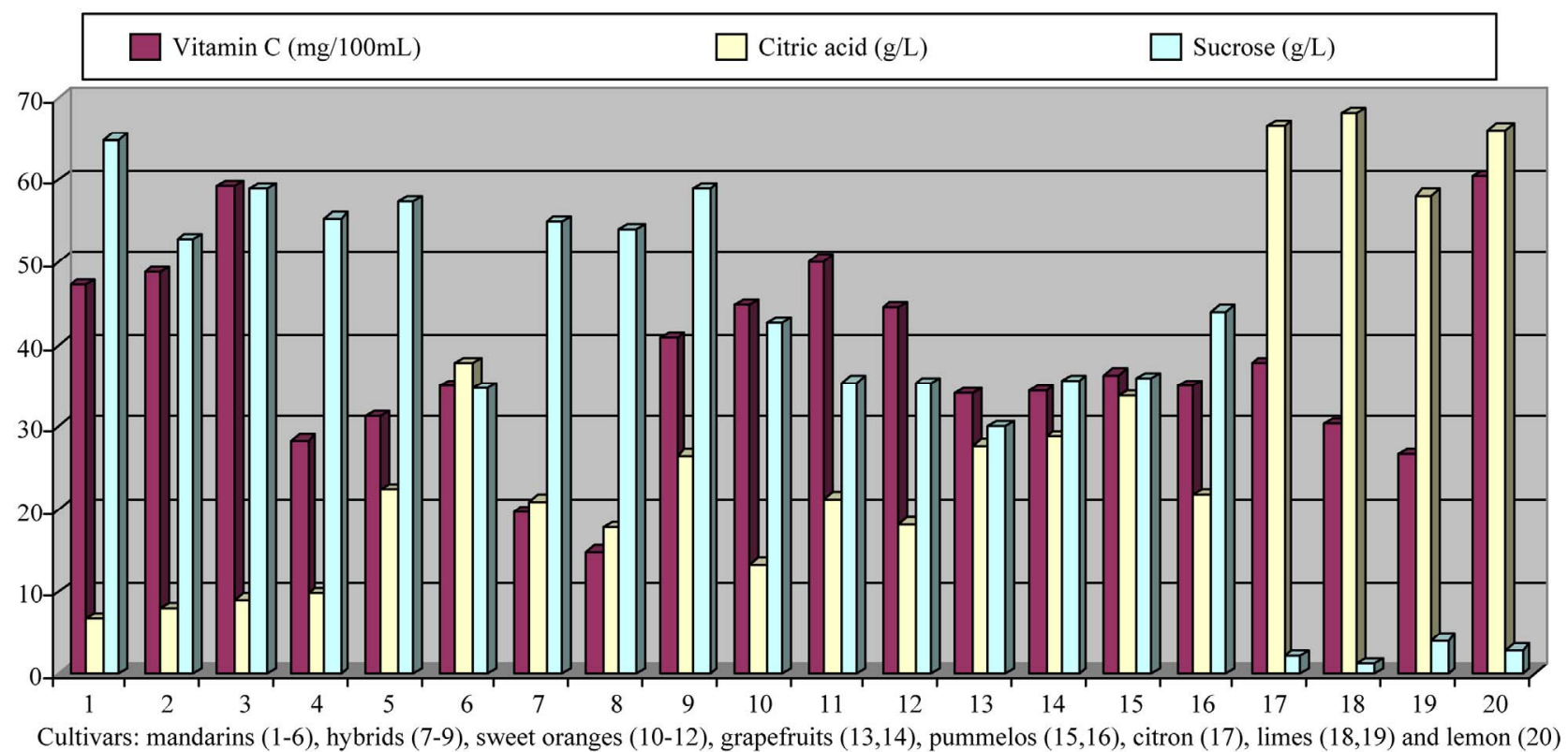

Figure 1. Vitamin C, citric acid and sucrose contents at commercial harvest time for various citrus fruit cultivars. 
Table 5. Changes in the maturity index, vitamin $\mathrm{C}$, organic acids and sugars for grapefruits and pummelos during maturation (from V to XI) ${ }^{x}$.

\begin{tabular}{|c|c|c|c|c|c|c|c|c|c|}
\hline \multirow{2}{*}{ Cultivar $^{1}$} & \multirow{2}{*}{ Date } & \multirow{2}{*}{ Mat. Index } & \multirow{2}{*}{ Vitamin $C^{2}$} & \multicolumn{3}{|c|}{ Organic acids (g/L juice) } & \multicolumn{3}{|c|}{ Sugars (g/L juice) } \\
\hline & & & & Citric & Malic & Succinic & Fructose & Glucose & Sucrose \\
\hline 13. "Marsh" & $\mathbf{V}$ & $4.52 \pm 0.09$ & $42.86 \pm 2.12$ & $25.57 \pm 0.44$ & $7.18 \pm 0.28$ & $0.58 \pm 0.07$ & $13.79 \pm 0.07$ & $13.83 \pm 0.31$ & $35.17 \pm 0.41$ \\
\hline 13. "Marsh" & VI & $4.43 \pm 0.47$ & $44.42 \pm 3.60$ & $26.05 \pm 1.58$ & $8.38 \pm 0.78$ & $0.87 \pm 0.20$ & $24.74 \pm 3.56$ & $24.06 \pm 1.32$ & $42.98 \pm 4.84$ \\
\hline 13. "Marsh" & VII & $4.20 \pm 0.22$ & $40.91 \pm 1.01$ & $26.87 \pm 0.22$ & $11.82 \pm 0.11$ & $0.85 \pm 0.04$ & $15.52 \pm 1.17$ & $15.36 \pm 0.26$ & $30.26 \pm 0.66$ \\
\hline 13. "Marsh" & VIII & $4.53 \pm 0.15$ & $32.88 \pm 2.05$ & $26.78 \pm 2.23$ & $8.25 \pm 0.09$ & $0.58 \pm 0.01$ & $17.15 \pm 0.36$ & $14.59 \pm 0.75$ & $31.23 \pm 4.82$ \\
\hline 13. "Marsh" & IX & $4.91 \pm 4.03$ & $36.77 \pm 0.39$ & $30.24 \pm 0.28$ & $11.24 \pm 0.03$ & $0.54 \pm 0.04$ & $17.49 \pm 0.48$ & $16.41 \pm 0.77$ & $33.48 \pm 1.51$ \\
\hline 13. "Marsh" & $\mathbf{X}$ & $5.01 \pm 0.57$ & $32.48 \pm 1.51$ & $26.46 \pm 0.49$ & $9.68 \pm 0.04$ & $0.65 \pm 0.01$ & $18.19 \pm 0.58$ & $17.26 \pm 0.58$ & $25.58 \pm 0.69$ \\
\hline 13. "Marsh" & XI & $5.17 \pm 0.10$ & $25.94 \pm 1.19$ & $19.21 \pm 0.79$ & $5.46 \pm 0.25$ & $0.53 \pm 0.01$ & $18.59 \pm 1.00$ & $17.54 \pm 0.54$ & $30.38 \pm 1.36$ \\
\hline 14. "Star Ruby" & $\mathbf{V}$ & $4.30 \pm 0.10$ & $53.14 \pm 2.02$ & $28.88 \pm 1.21$ & $7.59 \pm 0.48$ & $0.46 \pm 0.12$ & $10.71 \pm 0.49$ & $11.17 \pm 0.52$ & $36.47 \pm 1.64$ \\
\hline 14. "Star Ruby" & VI & $4.27 \pm 0.21$ & $45.84 \pm 2.36$ & $30.26 \pm 0.68$ & $6.64 \pm 0.17$ & $0.27 \pm 0.07$ & $15.95 \pm 3.28$ & $16.54 \pm 3.22$ & $34.35 \pm 5.47$ \\
\hline 14. "Star Ruby" & VII & $4.43 \pm 0.31$ & $42.75 \pm 3.05$ & $27.81 \pm 0.25$ & $5.96 \pm 0.33$ & $0.10 \pm 0.07$ & $13.15 \pm 0.77$ & $12.92 \pm 0.92$ & $28.68 \pm 1.74$ \\
\hline 14. "Star Ruby" & VIII & $4.48 \pm 0.37$ & $35.07 \pm 2.11$ & $30.80 \pm 2.16$ & $8.38 \pm 0.15$ & $0.32 \pm 0.13$ & $18.68 \pm 3.49$ & $15.93 \pm 3.19$ & $38.07 \pm 4.13$ \\
\hline 14. "Star Ruby" & IX & $4.91 \pm 0.09$ & $33.42 \pm 2.33$ & $28.47 \pm 0.50$ & $6.81 \pm 0.15$ & $0.26 \pm 0.04$ & $14.07 \pm 0.79$ & $13.55 \pm 0.35$ & $36.05 \pm 0.92$ \\
\hline 14. "Star Ruby" & $\mathbf{X}$ & $4.60 \pm 0.01$ & $34.42 \pm 0.60$ & $27.09 \pm 0.08$ & $6.46 \pm 0.05$ & $0.19 \pm 0.03$ & $16.08 \pm 0.40$ & $15.75 \pm 0.43$ & $32.47 \pm 0.90$ \\
\hline 14. "Star Ruby" & XI & $5.37 \pm 0.08$ & $36.93 \pm 2.84$ & $24.39 \pm 0.78$ & $7.37 \pm 0.27$ & $0.38 \pm 0.02$ & $15.77 \pm 1.21$ & $14.50 \pm 0.84$ & $36.41 \pm 2.24$ \\
\hline 15. “Gil” & $\mathbf{V}$ & $3.94 \pm 0.10$ & $49.90 \pm 1.85$ & $25.15 \pm 0.98$ & $10.26 \pm 3.81$ & $0.24 \pm 0.18$ & $7.81 \pm 0.41$ & $9.97 \pm 0.23$ & $35.57 \pm 0.76$ \\
\hline 15. “Gil” & VI & $3.82 \pm 0.36$ & $46.72 \pm 2.76$ & $33.12 \pm 0.33$ & $17.05 \pm 0.07$ & $0.40 \pm 0.02$ & $9.10 \pm 1.04$ & $9.89 \pm 0.60$ & $32.21 \pm 4.29$ \\
\hline 15. “Gil” & VII & $3.58 \pm 0.15$ & $44.14 \pm 0.10$ & $27.47 \pm 0.16$ & $8.50 \pm 0.04$ & $0.33 \pm 0.03$ & $7.40 \pm 0.05$ & $7.33 \pm 0.28$ & $32.79 \pm 0.85$ \\
\hline 15. “Gil” & VIII & $3.61 \pm 0.23$ & $32.16 \pm 1.96$ & $34.89 \pm 0.73$ & $10.41 \pm 0.19$ & $0.12 \pm 0.02$ & $13.00 \pm 0.92$ & $11.57 \pm 0.75$ & $31.33 \pm 3.67$ \\
\hline 15. “Gil” & IX & $4.37 \pm 0.13$ & $34.00 \pm 1.47$ & $33.59 \pm 0.56$ & $10.89 \pm 0.22$ & $0.24 \pm 0.01$ & $14.09 \pm 1.29$ & $15.47 \pm 0.94$ & $44.33 \pm 2.56$ \\
\hline 15. “Gil” & $\mathbf{X}$ & $4.26 \pm 0.01$ & $43.01 \pm 19.41$ & $32.98 \pm 0.28$ & $12.30 \pm 0.15$ & $0.15 \pm 0.01$ & $16.69 \pm 0.33$ & $16.77 \pm 0.13$ & $32.35 \pm 0.55$ \\
\hline 15. “Gil” & XI & $4.69 \pm 0.07$ & $32.32 \pm 0.49$ & $28.09 \pm 0.19$ & $15.15 \pm 0.18$ & $0.11 \pm 0.03$ & $17.67 \pm 0.65$ & $18.17 \pm 1.16$ & $30.56 \pm 0.88$ \\
\hline 16. “Deep Red” & $\mathbf{V}$ & $7.17 \pm 0.77$ & $56.32 \pm 3.74$ & $18.38 \pm 0.75$ & $10.25 \pm 0.21$ & $0.63 \pm 0.04$ & $10.32 \pm 0.77$ & $11.43 \pm 0.69$ & $42.12 \pm 3.60$ \\
\hline 16. "Deep Red" & VI & $5.97 \pm 0.86$ & $52.66 \pm 6.32$ & $17.71 \pm 0.08$ & $9.88 \pm 0.08$ & $0.42 \pm 0.05$ & $13.61 \pm 1.04$ & $15.01 \pm 0.55$ & $48.76 \pm 6.57$ \\
\hline 16. “Deep Red” & VII & $5.32 \pm 0.74$ & $56.58 \pm 13.67$ & $21.11 \pm 0.16$ & $9.81 \pm 0.08$ & $0.80 \pm 0.00$ & $11.55 \pm 0.11$ & $11.59 \pm 0.16$ & $41.17 \pm 0.15$ \\
\hline 16. “Deep Red” & VIII & $5.20 \pm 0.25$ & $34.32 \pm 1.66$ & $21.72 \pm 2.08$ & $8.35 \pm 1.35$ & $0.65 \pm 0.06$ & $15.33 \pm 4.09$ & $13.70 \pm 3.40$ & $54.74 \pm 12.29$ \\
\hline 16. "Deep Red" & IX & $5.38 \pm 0.60$ & $35.04 \pm 1.50$ & $22.83 \pm 0.92$ & $7.30 \pm 0.32$ & $0.64 \pm 0.03$ & $12.80 \pm 1.43$ & $12.67 \pm 2.77$ & $40.61 \pm 0.96$ \\
\hline 16. "Deep Red" & $\mathbf{X}$ & $5.15 \pm 0.01$ & $35.41 \pm 0.82$ & $21.06 \pm 0.32$ & $8.31 \pm 0.20$ & $0.54 \pm 0.04$ & $10.28 \pm 0.53$ & $10.13 \pm 0.28$ & $36.89 \pm 1.08$ \\
\hline 16. “Deep Red” & XI & $6.17 \pm 0.05$ & $41.20 \pm 2.99$ & $20.14 \pm 0.91$ & $11.37 \pm 0.67$ & $0.72 \pm 0.05$ & $9.66 \pm 0.73$ & $8.85 \pm 0.44$ & $40.37 \pm 2.40$ \\
\hline
\end{tabular}

${ }^{\mathrm{x}}$ Data are expressed as mean (maturity index and vitamin C: $\mathrm{n}=6$; organic acids and sugars: $\mathrm{n}=3$ ) \pm standard deviation; ${ }^{1}$ Rootstocks: Troyer; ${ }^{2} \mathrm{mg}$ vitamin $\mathrm{C}$ total/100mL juice.

2009 and March 22, 2010, maintaining or improving their fruit quality, although not all cultivars can be kept so long in the tree. Our results indicated differences between rootstocks, although it will be necessary to carry out more in-depth analysis to report conclusive values. Considering the maturity index values for each harvest time, we found significant differences between cultivars grafted onto Troyer citrange and Cleopatra mandarin (Table 2). "Loretina" and "Frost" mandarins, "Fortune" hybrid, "Navelate" and "Valencia Late" oranges, "Arizona" citron and, "Mejicana" and "Bearss" limes grafted onto Troyer citrange displayed the highest values of maturity index compared with the corresponding cultivars grafted onto Cleopatra mandarin. Also we found significant differences between the maturity index of cultivars of "Fino" lemon grafted both onto C. macrophylla and $C$. aurantium. The results are in agreement with those reported in the literature observing the effects of citrus rootstoks on internal quality and matutity [6,7].

Citrus are well known to be a nutrient source of vitamin $\mathrm{C}$ in dietary intake. Data of total ascorbic acid for each harvest time are reported in milligrams per $100 \mathrm{~mL}$ of juice. The general trend in all varieties studied was a decrease in the concentration of vitamin $\mathrm{C}$ over time, and significant differences were observed in all varieties, being particularly noteworthy in the case of grapefruits 
Table 6. Changes in the maturity index, vitamin C, organic acids and sugars for citron, limes and lemon during maturation (from V to XI)

\begin{tabular}{|c|c|c|c|c|c|c|c|c|c|}
\hline \multirow{2}{*}{ Cultivar $^{1}$} & \multirow{2}{*}{ Date } & \multirow{2}{*}{ Mat. Index } & \multirow{2}{*}{ Vitamin $C^{2}$} & \multicolumn{3}{|c|}{ Organic acids (g/L juice) } & \multicolumn{3}{|c|}{ Sugars (g/L juice) } \\
\hline & & & & Citric & Malic & Succinic & Fructose & Glucose & Sucrose \\
\hline 17. “Arizona” & $\mathbf{V}$ & $1.17 \pm 0.03$ & $38.94 \pm 4.59$ & $65.20 \pm 1.63$ & $9.26 \pm 0.11$ & $0.29 \pm 0.10$ & $2.43 \pm 0.23$ & $2.51 \pm 0.08$ & $2.31 \pm 0.20$ \\
\hline 17. “Arizona” & VI & $1.06 \pm 0.00$ & $32.50 \pm 5.58$ & $67.89 \pm 1.15$ & $8.58 \pm 0.11$ & $0.47 \pm 0.04$ & $2.02 \pm 0.16$ & $2.05 \pm 0.32$ & $1.41 \pm 0.32$ \\
\hline 17. “Arizona” & VII & $1.10 \pm 0.03$ & $29.98 \pm 5.26$ & $66.51 \pm 0.73$ & $7.30 \pm 0.10$ & $0.51 \pm 0.06$ & $2.87 \pm 0.31$ & $2.60 \pm 0.22$ & $2.90 \pm 0.29$ \\
\hline 17. “Arizona” & VIII & $1.12 \pm 0.05$ & $20.80 \pm 0.70$ & $62.47 \pm 0.67$ & $8.76 \pm 0.35$ & $0.54 \pm 0.01$ & $2.89 \pm 0.21$ & $2.33 \pm 0.22$ & $3.53 \pm 0.38$ \\
\hline 17. “Arizona” & IX & $1.50 \pm 0.02$ & $20.61 \pm 1.49$ & $47.25 \pm 1.30$ & $6.94 \pm 0.13$ & $0.50 \pm 0.03$ & $5.19 \pm 0.31$ & $3.79 \pm 0.19$ & $5.20 \pm 0.34$ \\
\hline 18. "Mejicana” & $\mathbf{V}$ & $1.10 \pm 0.03$ & $28.19 \pm 2.21$ & $66.52 \pm 2.26$ & $10.86 \pm 0.09$ & $0.95 \pm 0.11$ & $1.11 \pm 0.06$ & $1.32 \pm 0.24$ & $1.17 \pm 0.05$ \\
\hline 18. “Mejicana” & VI & $1.08 \pm 0.02$ & $28.32 \pm 0.93$ & $69.63 \pm 1.78$ & $11.64 \pm 0.04$ & $1.22 \pm 0.03$ & $1.36 \pm 0.09$ & $1.51 \pm 0.20$ & $1.40 \pm 0.28$ \\
\hline 19. "Bears" & $\mathbf{V}$ & $1.53 \pm 0.05$ & $29.39 \pm 2.56$ & $56.38 \pm 0.18$ & $12.36 \pm 0.25$ & $0.23 \pm 0.02$ & $8.32 \pm 0.15$ & $9.12 \pm 0.29$ & $4.01 \pm 0.14$ \\
\hline 19. "Bears" & VI & $1.41 \pm 0.08$ & $28.53 \pm 2.85$ & $60.63 \pm 2.11$ & $13.10 \pm 0.19$ & $0.38 \pm 0.03$ & $4.64 \pm 0.75$ & $5.80 \pm 1.20$ & $3.31 \pm 0.36$ \\
\hline 19. "Bears" & VII & $1.38 \pm 0.03$ & $23.06 \pm 2.85$ & $57.51 \pm 0.82$ & $13.55 \pm 0.22$ & $0.21 \pm 0.02$ & $9.74 \pm 1.23$ & $10.37 \pm 0.94$ & $5.05 \pm 0.76$ \\
\hline 19. "Bears" & VIII & $1.26 \pm 0.01$ & $20.83 \pm 2.09$ & $60.04 \pm 1.66$ & $13.57 \pm 0.63$ & $0.20 \pm 0.04$ & $7.80 \pm 1.74$ & $9.29 \pm 0.63$ & $5.41 \pm 0.69$ \\
\hline 19. "Bears" & IX & $1.40 \pm 0.03$ & $18.32 \pm 1.11$ & $55.14 \pm 0.19$ & $11.10 \pm 0.07$ & $0.20 \pm 0.01$ & $6.31 \pm 0.74$ & $5.13 \pm 0.59$ & $3.35 \pm 0.09$ \\
\hline 20. “Fino" & V & $1.27 \pm 0.06$ & $65.18 \pm 1.56$ & $65.02 \pm 2.26$ & $14.65 \pm 0.37$ & $0.40 \pm 0.01$ & $3.77 \pm 0.32$ & $4.50 \pm 0.48$ & $2.56 \pm 0.25$ \\
\hline 20. "Fino" & VI & $1.17 \pm 0.08$ & $60.13 \pm 3.03$ & $67.01 \pm 3.33$ & $16.68 \pm 0.49$ & $0.22 \pm 0.01$ & $4.22 \pm 0.48$ & $4.80 \pm 0.55$ & $3.46 \pm 0.31$ \\
\hline 20. "Fino" & VII & $1.18 \pm 0.02$ & $56.22 \pm 7.47$ & $66.39 \pm 0.36$ & $15.50 \pm 1.25$ & $0.34 \pm 0.02$ & $3.29 \pm 0.11$ & $3.02 \pm 0.13$ & $2.84 \pm 0.06$ \\
\hline 20. "Fino" & VIII & $1.28 \pm 0.03$ & $44.91 \pm 1.91$ & $67.90 \pm 0.41$ & $12.35 \pm 0.47$ & $0.74 \pm 0.04$ & $7.04 \pm 0.14$ & $5.99 \pm 0.46$ & $9.29 \pm 0.77$ \\
\hline 20. "Fino" & IX & $1.43 \pm 0.01$ & $47.84 \pm 3.20$ & $65.67 \pm 1.89$ & $17.24 \pm 0.41$ & $0.32 \pm 0.03$ & $4.53 \pm 0.29$ & $4.91 \pm 0.22$ & $7.40 \pm 0.46$ \\
\hline 20. “Fino" & $\mathbf{X}$ & $1.30 \pm 0.01$ & $40.61 \pm 4.07$ & $63.06 \pm 1.52$ & $17.21 \pm 0.63$ & $0.51 \pm 0.03$ & $4.36 \pm 0.41$ & $4.33 \pm 0.12$ & $5.90 \pm 0.63$ \\
\hline
\end{tabular}

${ }^{\mathrm{x}}$ Data are expressed as mean (maturity index and vitamin C: $\mathrm{n}=6$; organic acids and sugars: $\mathrm{n}=3$ ) \pm standard deviation; ${ }^{1}$ Rootstocks: Troyer for $\mathbf{1 7}-\mathbf{1 9}$, Macrophylla for $20 ;{ }^{2} \mathrm{mg}$ vitamin $\mathrm{C}$ total $/ 100 \mathrm{~mL}$ juice.

and pummelos. The results are in agreement with those reported in the literature observing that inmature citrus fruits contain the highest concentration of vitamin C [9]. Considering the vitamin $\mathrm{C}$ values for each harvest time, we also found significant differences between cultivars grafted on different rootstocks (Table 2). The general trend was a higher vitamin $\mathrm{C}$ content in all varieties studied grafted on Troyer citrange. Only "Loretina" mandarin and "Navelate" orange grafted on Cleopatra mandarin showed higher vitamin $\mathrm{C}$ content that corresponding cultivars grafted on Troyer citrange. Also we found significant differences between the cultivars of "Fino" lemon grafted both onto C. macrophylla and C. aurantium (sour orange). At commercial harvest stage, independently of rootstocks, "Fino" lemon displayed the highest concentration of vitamin C $(60.51 \mathrm{mg} / 100 \mathrm{~mL}$ juice $)$ followed by clementine mandarins (59.30 to $47.26 \mathrm{mg} / 100 \mathrm{~mL}$ juice) and sweet oranges (50.22 to $44.57 \mathrm{mg} / 100 \mathrm{~mL}$ juice), although during the 2009-2010 season also the grapefruits and pummelos showed high values (see Figure 1 and Table 3). These results are in agreement with previously reported citrus study of several mandarin and orange varieties $[1,15]$. Our results indicated significative differences between all cultivars studied. Between groups, the vitamin $\mathrm{C}$ content of clementine mandarin "Arrufatina" differed significantly to "Loretina" and "Fina" clementines. In the group of oranges found no significant differences, nor among the grapefruits, and nor among pummelos.

Three organic acids were separated and identified in all cultivars: citric, malic and succinic acid. The amounts of each organic acid found from November 02, 2009 (V) to March 22, 2010 (XI) are reported in grams per L of juice, and only grafted on one rootstocks (see Tables 4-6). As indicated by previous researchers, citric acid was the major organic acid found in all cultivars, while malic and succinic acids were present in minor quantities [8]. The general trend in all varieties studied is a decrease in the concentration of the organic acids over time from the best period time for harvest, and signicant differences were observed. At commercial harvest stage during the 2009-2010 season, independently of rootstocks, citron "Arizona", limes "Mejicana" and "Bearss", and lemon "Fino" were the most acidic fruit reaching maximum values (68.08 to $58.17 \mathrm{~g}$ citric acid/L) compared with the remaining cultivars studied, and clementine mandarins displayed the least amounts (6.68 to $9.06 \mathrm{~g}$ citric acid/L) (Figure 1). Our results indicated significative differences 
between all cultivars studied. Between groups, the citric acid content of the sweet orange "Navelate" differed significanthly to "Valencia Late" and "Sanguinelli" oranges. Among pummelos we also observed significant differences. In the group of grapefruits found no significant differences, nor among the clementine mandarins.

The main portions of carbohydrates in citrus fruits are three simple sugars: fructose, glucose and sucrose, they represent the largest percentage of total soluble solids of citrus juice, and the ratios of fructose:glucose:sucrose are generally about 1:1:2 [8]. Except in citrons, limes and lemons with the least amount of sugars, this ratio was similar for the cultivars under study, and sucrose was present in the largest amounts for all cultivars. The general trend in all varieties studied is a significant increase in the concentration of the sugars over time (see Tables 4-6). At commercial harvest stage, independently of rootstocks, mandarins (except "Comun" mandarin) and hybrids groups presented the highest amounts (64.88 to $52.88 \mathrm{~g}$ sucrose/L). Our results indicated not significative differences between similar groups (Figure 1).

As it is shown in Figure 1, a high load of citric acid appeared in acidic citrus fruits, and a high load of sucrose and/or total vitamin $\mathrm{C}$ appeared in oranges and mandarins. Although our results indicated significative differences between cultivars studied, it is apparent that clementine mandarins (cultivars 1-3) formed a single group and sweet oranges (cultivars 10-12) form a second group. The following group are formed by grapefruits and pumelos (cultivars 13-16) and the last group are formed by citron and limes (cultivars 17-19). Outside these four groups appear the remaining cultivars (hybrids, lemon and other mandarins). For nutritional purpose, would be advisable a more intensive study on the other antioxidant compounds in the edible part of Citrus species (major flavonoids and carotenoids), but data presented in this paper confirmed the high contents of vitamin $\mathrm{C}$ in citrus varieties cultived under the Mediterranean climate and provide a qualitative and quantitative survey of the fruit taste and organoleptic quality. These aspects are necessary for the consumer that demands the prevention of health problems through nutrition and certain fruit quality traits including fruit size, internal quality, good rind colour and easy peeling.

\section{Conclusion}

In this study, we have presented the content in total vita$\min \mathrm{C}$, organic acids and sugars for twenty different citrus cultivars grown in the Mediterranean climate during 2009-2010 season. We have examined the influence of the variety, rootstocks and different stages of fruit maturity. The fruit quality was affected differently and we have found clear differences in accordance with the root- stocks but, the major differences in chemical composition must be attributed mainly to genetic factors. We have found clear differences in the content of sugars, ascorbic and organic acids for the different groups, in agreement with the Citrus classification with some exception. Also climatic and cultural factors have affected to fruit quality, and anticipate or delay the collection generally results in a loss of bioactive compounds. On the other hand, the data presented are an important factor to chose varieties with a high potential as nutraceutical source.

\section{Acknowledgements}

This work was supported by research project RTA200800052-00-00 from INIA (Ministerio de Educación y Ciencia, Spain), and the financial help of the European Community (FEDER and ESF funds) and Consellería de Agricultura, Pesca y Alimentación (Generalitat Valenciana, Spain). I thank Alejandro Medina from the Field Collection of Citrus Germoplasm Bank (IVIA) for his assistence during the preparation of this manuscript.

\section{REFERENCES}

[1] C. Dhuique-Mayer, C. Caris-Veyrat, P. Ollitrault, F Curck and M. J. Amiot, "Varietal and Interspecific Influence on Micronutrient Contents in Citrus from the Mediterranean Area," Journal of Agricultural and Food Chemistry, Vol. 53, No. 6, 2005, pp. 2140-2145. doi:10.1021/jf0402983

[2] M. A. Germanà, V. Mineo and B. Chiancone, "Study on Flavonoid Contents in Fruits of Different of Citrus Genotypes", Acta Horticulturae (ISHS), Vol. 632, No. 1, 2004, pp. 355-360.

[3] H. Merle, M. Morón, M. A. Blázquez and H. Boira, "Taxonomical Contribution of Essencial Oils in Mandarins Cultivars," Biochemical Systematics and Ecology, Vol. 32, No. 5, 2004, pp. 491-497.

doi:10.1016/j.bse.2003.09.010

[4] Y. Nogata, K. Sakamoto, H. Shiratsuchi, T. IshiI, M. Yano and H. Ohta, "Flavonoid Composition of Fruit Tissues of Citrus Species," Bioscience, Biotechnology, and Biochemistry, Vol. 70, No. 1, 2006, pp. 178-192. doi:10.1271/bbb. 70.178

[5] M. V. Albertini, E. Carcouet, O. Pailly, C. Gambotti, F. Luro and L. Berti, "Changes in Organic Acids and Sugars during Early Stages of Development of Acidic and Acidless Citrus Fruit," Journal of Agricultural and Food Chemistry, Vol. 54, No. 21, 2006, pp. 8335-8339. doi:10.1021/jf061648j

[6] M. Agustí, "Patrones," Citricultura, 2nd Edition, MundiPrensa, Madrid, 2003, pp. 331-349.

[7] W. S. Castle, "Review: Rootsock as a Fruit Quality Factor in Citrus and Deciduous Tree Crops," New Zealand Journal of Crop and Horticultural Science, Vol. 23, No. 4, 
1995, pp. 383-394

doi:10.1080/01140671.1995.9513914

[8] H. Kelebek, S. Selli, A. Canbas and T. Cabaroglu, "HPLC Determination of Organic Acids, Sugars, Phenolic Compositions and Antioxidant Capacity of Orange Juice and Orange Wine Made from a Turkish cv. Kozan," Microchemical Journal, Vol. 91, No. 2, 2009, pp. 187-192. doi:10.1016/i.microc.2008.10.008

[9] S. K. Lee and A. A. Kader, "Preharvest and Postharvest Factors Influencing Vitamin C Content of Horticultural Crops," Postharvest Biololy and Technology, Vol. 20, No. 3, 2000, pp. 207-220. doi:10.1016/S0925-5214(00)00133-2

[10] F. Karadeniz, "Main Organic Acid Distribution of Authentic Citrus Juices in Turkey," Turkish Journal of Agriculture and Forestry, Vol. 28, No. 4, 2004, pp. 267-271.

[11] V. Nour, I. Trandafir and M. E. Ionica, "HPLC Organic Acid Analysis in Different Citrus Juices under Reverserd Phase Conditions," Notulae Botanicae Horti Agrobotanici Cluj-Napoca Vol. 38, No. 1, 2010, pp. 44-48.

[12] K. V. R. Raman, V. S. Govindarajan and S. Ranganna, "Citrus Fruits-Varieties, Chemistry, Technology, and Quality Evaluation. I. Varieties, Production, Handling, and Storage," CRC Critical Reviews in Food Science and Nutrition, Vol. 15, No. 4, 1981, pp. 353-431. doi:10.1080/10408398109527321

[13] S. Ranganna, V. S. Govindarajan and K. V. R. Ramana, "Citrus Fruits-Varieties, Chemistry, Technology, and Quality Evaluation. II. Chemistry, Technology, and Quality Evaluation. A. Chemistry," CRC Critical Reviews in
Food Science and Nutrition, Vol. 18, No. 4, 1983, pp. 313-386. doi:10.1080/10408398309527366

[14] A. Bermejo, J. Pardo and A. Cano, "Influence of Gamma Irradiation on Seedless Citrus Production: Pollen Germination and Fruit Quality," Food and Nutrition Sciences, Vol. 2, No. 3, 2011, pp. 169-180. doi:10.4236/fns.2011.23024

[15] A. Cano, A. Medina and A. Bermejo, "Bioactive Compounds in Different Citrus Varieties. Discrimination among Cultivars," Journal of Food Composition and Analysis, Vol. 21, No. 5, 2008, pp. 377-381. doi:10.1016/j.jfca.2008.03.005

[16] A. Cano and A. Bermejo, "Influence of Rootstock and Cultivar on Bioactive Compounds in Citrus Peels," Journal of the Science of Food and Agriculture, Vol. 91, No. 9, 2011, pp. 1702-1711. doi:10.1002/jsfa.4375

[17] O. Pailly, G. Ison and A. Amouroux, "Harvest Time and Storage Conditions of 'Star Ruby' Grapefruit (Citrus paradisi Macf.) for Short Distance Consumption," Postharvest Biololy and Technology, Vol. 34, No. 1, 2004, pp. 65-73.

[18] M. S. Ladaniya and B. C. Mahalle, "Fruit Maturation and Associated Changes in 'Mosambi' Orange (Citrus sinensis)," Indian Journal of Agricultural Sciences, Vol. 81, No. 6, 2011, pp. 494-499.

[19] P. N. Kale and P. G. Adsule, "Handbook of Fruit Science and Technology. Production, Composition, Storage and Processing," In: D. K. Salunkhe and S. S. Kadam, Eds., Marcel Dekker, New York, 1995. 